\title{
Human acid-insoluble dentin with BMP-2 accelerates bone induction in subcutaneous and intramuscular tissues
}

\author{
Masaru MURATA, ${ }^{\dagger}$ Tatsushi KAWAI, ${ }^{*}$ Toshiyuki KAWAKAMI, ${ }^{* *}$ Toshiyuki AKAZAWA, ${ }^{* * *}$ \\ Junichi TAZAKI, Katsutoshi ITO, Kaoru KUSANO and Makoto ARISUE \\ Oral and Maxillofacial Surgery, School of Dentistry, Health Sciences University of Hokkaido, \\ 1757 Kanazawa, Tobetsu, Hokkaido 061-0293 \\ *Dental Material Science, School of Dentistry, Aichi-Gakuin University, 1-100 Kusumoto-cho, Chikusa-ku, Nagoya, Aichi $464-8650$ \\ ** Institute for Oral Science \& High-Tech Center, Matsumoto Dental University, 1780 Hirokagouhara, Shiojiri, Nagano 399-0781 \\ ***Materials Technology, Hokkaido Industrial Research Institute, Kita 19 Nishi 11, Kita-ku, Sapporo, Hokkaido $060-0819$
}

\begin{abstract}
Dentin matrix is well known as the most insoluble collagen in human body. We already confirmed the osteoinductive property by granules of human demineralized dentin matrix (DDM) histologically. Human DDM granules and human demineralized rootdentin (DRD) mass were prepared from adult vital teeth. The hard tissue induction by DDM alone or DRD alone was estimated at 4 weeks after implantation. The DDM alone induced bone and cartilage independently. In addition, the bioassay by recombinant human BMP-2 $(5.0 \mu \mathrm{g}) / \mathrm{DDM}$ or BMP-2 $(5.0 \mu \mathrm{g}) / \mathrm{DRD}$ was estimated in mouse subcutaneous or intramuscular tissues, respectively. Histological examination showed that the BMP-2/DDM induced bone and marrow, and the DDM granules were partially absorbed by new bone. The morphometric analysis demonstrated that the BMP-2/DDM showed $36.3 \%$ in the volume of bone and marrow, while the DDM alone showed $1.3 \%$ at 4 weeks. Moreover, the BMP-2/DRD also induced active bone formation on the root surface and in the pulp cavity. These results indicate that BMP-2 significantly accelerated bone formation in decalcified dentin implants. Human recycled DDM and DRD might be effective materials as osteoinductive collagenous carriers of BMP-2 for bone engineering.
\end{abstract}

(C2010 The Ceramic Society of Japan. All rights reserved.

Key-words : Human, Dentin, Demineralization, Bone Induction, BMP-2

[Received February 9, 2010; Accepted April 15, 2010]

\section{Introduction}

Dentin is an acellular collagen-rich tissue matrix without vessels, while bone is a cellular tissue with vessels. However, dentin and bone have similar components that consist of $10 \%$ body fluid, $20 \%$ organic materials, and $70 \%$ minerals as mainly hydroxyapatite (HAp), and contain bone morphogenetic proteins (BMPs) with inducing capability of bone and cartilage in nonskeletal sites. ${ }^{1)}$ Recombinant human BMP-2, 4, and 7 are strong accelerating factors of bone induction, ${ }^{2), 3)}$ and BMP-2 and 7 are available for clinical use in USA and European Union.

The bone-inducing property of dentin was discovered in 1967 that rabbit demineralized dentin matrics (DDM) induced bone in the intramuscular tissues. ${ }^{4)}$ There are several in vivo studies that the animal DDM induced ectopic bone formation in subcutaneous and intramuscular pockets in rodents. ${ }^{5,6)}$ We confirmed histologically that human completely demineralized dentin granules induced bone and cartilage independently in nude mice.7),8) $\mathrm{On}$ the other hand, human partially demineralized dentin granules failed to induce bone formation in the intramuscular pockets. ${ }^{9)}$ These reports indicated that dentin preparation such as shape and acid-treatment conditions was critical for bone-inducing property of dentin. Though DDM and DRD are defined as acid-insoluble collagen binding natural BMPs, we considered human recycled DDM and DRD as absorbable biomaterials for the better performances of recombinant human BMP-2.

\footnotetext{
Corresponding author: M. Murata; E-mail: murata@hoku-iryo-u. ac.jp
}

The purpose of this study is to estimate the efficacy of the granule type and the root type of human completely demineralized dentin as carrier matrics for the BMP-2 delivery system.

\section{Experimental procedure}

\subsection{Experiment 1}

\subsubsection{Preparation of dentin granules}

The human, adult third molar teeth were crushed under the ice cooling, using our developed auto-mill with $\mathrm{ZrO}_{2}$ vessel and blade (Figs. 1, 2), and completely demineralized in $2 \% \mathrm{HNO}_{3}$ solution (pH 1.0) for DDM (Figs. 1-3). The acid-insoluble DDM granules were sieved (size: $0.5-1.0 \mathrm{~mm}$ ), lyophilized and reserved in $-80^{\circ} \mathrm{C}$ before use. The insoluble dentin collagen is not expanded in $\mathrm{HCl}$ solution ( $\mathrm{pH} 2.0$ ), while the expansion rates of skin collagen and bone collagen are about 4.0 and 1.2 times, respectively. ${ }^{10)}$

\subsubsection{Addition of BMP-2 solution}

One hundred micro-liter of recombinant human BMP-2 $(50 \mu \mathrm{g} / \mathrm{ml}$ PBS $)$ solution was added by pipetting to $70 \mathrm{mg}$ of DDM in a cut-opened tuberculin syringe $\left(1 \mathrm{ml}\right.$, TERUMO $\left.{ }^{\circledR}\right)$ just before the operations. Recombinant human BMP-2 was supplied by Astellas Pharma Inc. (Tokyo, Japan), and was prepared at a concentration of $0.1 \mu \mathrm{g}$ of BMP-2 per microliter of phosphate buffered saline (PBS).

\subsubsection{Bioassay}

Nude mice (male, 4 week-old), deficient in immunogenic reactions, were subjected to intraperitoneal anesthesia with pentobarbital sodium $(4 \mathrm{mg} / 100 \mathrm{~g}$ body weight). BMP-2 $(5.0 \mu \mathrm{g}) / \mathrm{DDM}(70 \mathrm{mg})$ were implanted into the subcutaneous 


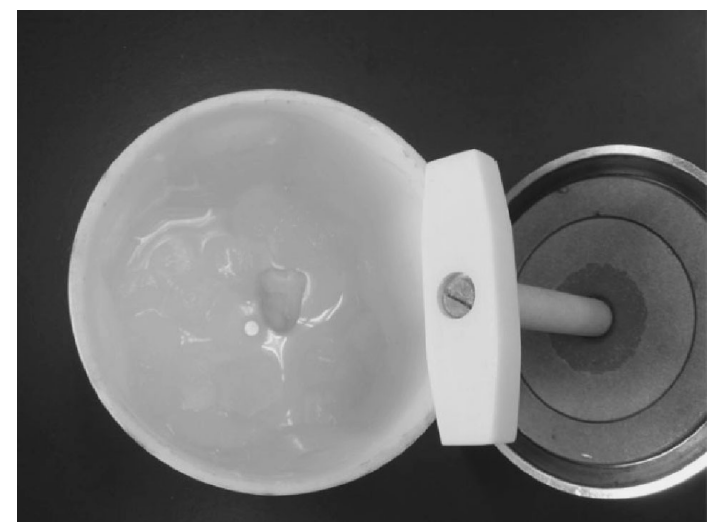

Fig. 1. Extracted tooth with 20 ices in $\mathrm{ZrO}_{2}$ vessel before crushing.

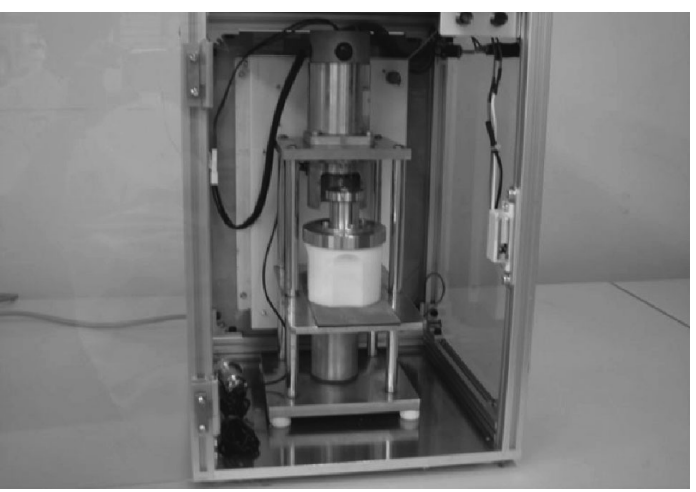

Fig. 2. Automatic mill. Newly developed mill (Patents: International application No. PCT/JP2007/053321, International published No. WO2007/099861 A1).

tissues in back. The incisions were sutured with nylon threads. The implanted materials were removed at 4 weeks after the operations. All procedures were followed the Guidelines in Health Sciences University of Hokkaido for Experiments on Animals.

\subsubsection{Histomorphological examinations}

The explanted samples were fixed in $10 \%$ neutral buffered formalin for 3 days, decalcified with decalcified for 7 days with $10 \%$ formic acid and embedded in paraffin. Decalcified sections ( $4 \mu \mathrm{m}$ in thickness) were made and stained with hematoxylin and eosin (HE). For morphometric analysis, tissues of the specimens were divided into five compartments: bone, cartilage, marrow, dentin matrix (DDM), and connective tissue. The compartments were measured by using Weibel method ${ }^{11)}$ at 3 separate points, $100 \mu \mathrm{m}$ apart, the midpoint being at center of the implant. The average value of these 3 points was used as the mean area of each implant.

\subsection{Experiment 2}

\subsubsection{Preparation of root-dentin}

Crowns were cut from human teeth and roots were collected. The roots were completely decalcified in $2 \% \mathrm{HNO}_{3}$ solution (pH 1.0) for demineralized root-dentin (DRD). The acid-insoluble DRD were lyophilized and reserved in $-80^{\circ} \mathrm{C}$ before use.

\subsubsection{Addition of BMP-2 solution}

One hundred micro-liter of recombinant human BMP-2 $(50 \mu \mathrm{g} / \mathrm{ml}$ PBS $)$ solution was added by pipetting to DRD in a sterilized tube just before the operations.

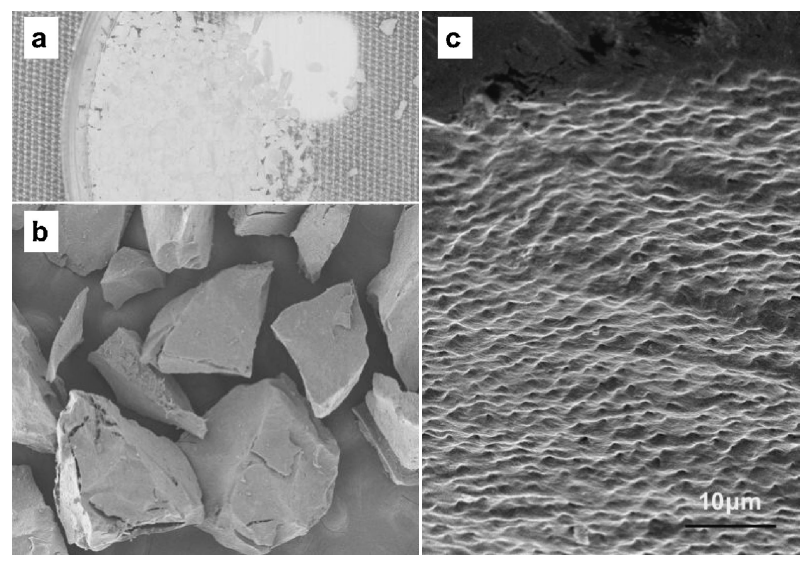

Fig. 3. Aspects of DDM granules. a. freeze-dried DDM before graft, b. SEM of DDM, c. higher magnification of b. Note: Dentinal tubes.

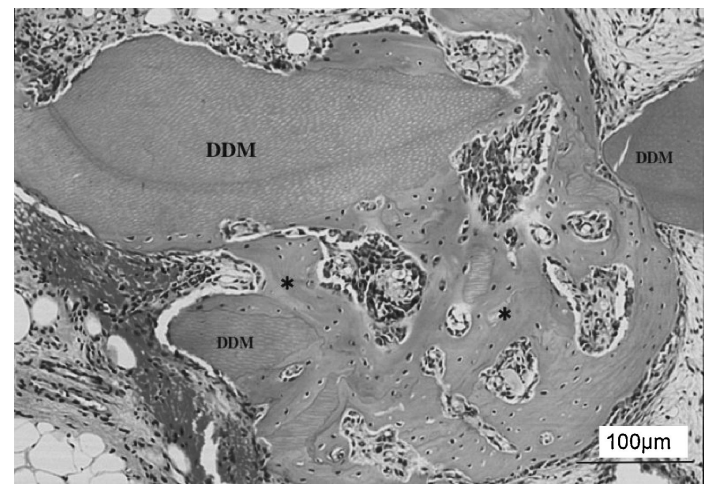

Fig. 4. Histological photograph of decalcified section of BMP-2/DDM at 4 weeks. ${ }^{*}$ induced bone (HE).

\subsubsection{Bioassay}

BMP-2 $(5.0 \mu \mathrm{g}) / \mathrm{DRD}$ were implanted into the hindquarter muscles in the nude mice (male, 4 week-old). At 4 weeks after the operations, the legs including implants were amputated and fixed with $10 \%$ neutral buffered formalin, decalcified for 10 days with $10 \%$ formic acid and embedded in paraffin. Decalcified sections $(4 \mu \mathrm{m}$ in thickness) were made and stained with hematoxylin and eosin (HE). All procedures were followed the Guidelines in Aichi-Gakuin University for Experiments on Animals.

\subsubsection{Radiography and histological examinations}

The samples were photographed by X-ray machine $(25 \mathrm{~mA}$, $24 \mathrm{~s}$ ), and then fixed in $10 \%$ neutral buffered formalin, decalcified with $10 \%$ formic acid, embedded in paraffin, sectioned and stained with HE.

\section{Results}

\subsection{Experiment 1}

\subsubsection{Histological findings}

In the BMP-2/DDM, osteoblast differentiation occurred on the surface of DDM, while chondroblast differentiation was not observed at 4 weeks (Fig. 4). Giant cells appeared on DDM surface and the absorption of DDM proceeded from the surfaces (Fig. 5). In DDM alone, new bone was found on the surface of DDM, and cartilage was observed at 4 weeks between DDM granules.

\subsubsection{Histomorphometrical analysis}

The morphometric results are shown in Table 1. The BMP-2/ 


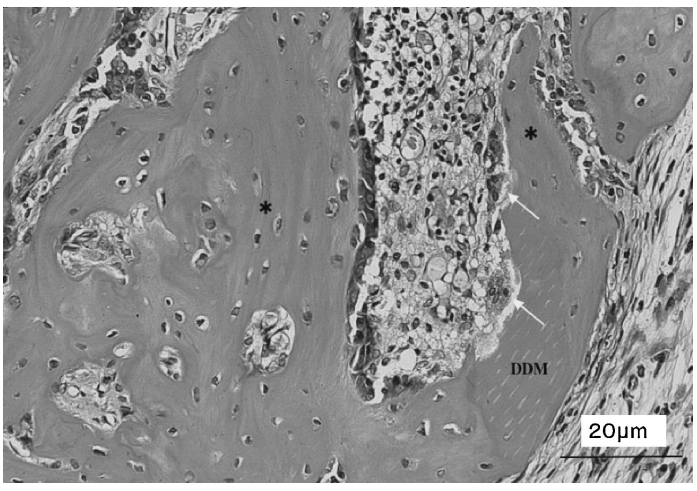

Fig. 5. Histological photograph of decalcified section of BMP-2/DDM at 4 weeks. $\uparrow$ giant cell on DDM. ${ }^{*}$ Induced bone (HE).

Table 1. Morphometric analysis (\%)

\begin{tabular}{lrcccc}
\hline Implant & Bone & Cartilage & Marrow & DDM & $\begin{array}{c}\text { Connective } \\
\text { tissue }\end{array}$ \\
\hline DDM alone & $0.9 \pm 0.1$ & $0.4 \pm 0.2$ & $0.4 \pm 0.1$ & $74.7 \pm 4.9$ & $23.6 \pm 5.0$ \\
DDM/BMP-2 & $28.3 \pm 2.6$ & 0 & $8.0 \pm 2.9$ & $34.4 \pm 2.9$ & $29.3 \pm 2.1$ \\
\hline
\end{tabular}

All tissues: $100 \%$, Value: mean \pm SD

Number: 3-9 implants, Explanted time: 4 weeks

DDM implant showed $36.3 \%$ at 4 weeks in the total volume of bone and marrow, while the DDM alone implant showed $1.3 \%$. The BMP-2/DDM showed $0.0 \%$ in cartilage, while the DDM alone implant showed $0.4 \%$. The ratio of DDM residues in the BMP-2/DDM was $67.5 \%$ of that in the DDM alone.

\subsection{Experiment 2}

\subsubsection{X-ray findings}

In the BMP-2/DRD, bone-like radiopaque was observed around DRD, while calcified tissues were not found in the DRD alone (Fig. 6).

\subsubsection{Histological findings}

In the BMP-2/DRD, active bone formation was observed around DRD and in micro-cracks at 4 weeks (Fig. 7). Mesenchymal cells invaded into the micro-cracks and pulp spaces. DRD mass was incorporated by trabecular bone and marrow (Fig. 8). Giant cells phagocyted DRD matrix, partially. In the DRD alone, bone and cartilage were not observed.
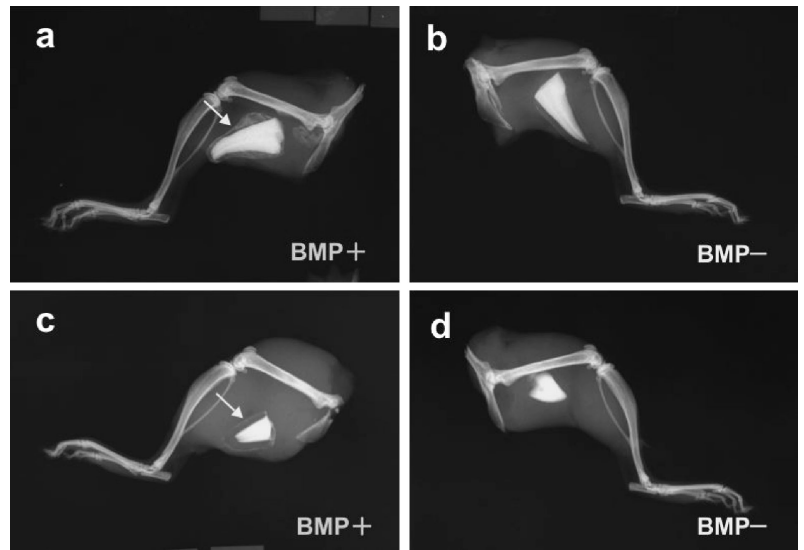

Fig. 6. Radiograph of intramuscular implants of BMP-2/DRD and DRD alone at 4 weeks. a, c: BMP-2/DRD, $\uparrow$ hard tissue-like radiopacity; b, d: DRD alone; a, b: same mouse; c, d: same mouse.

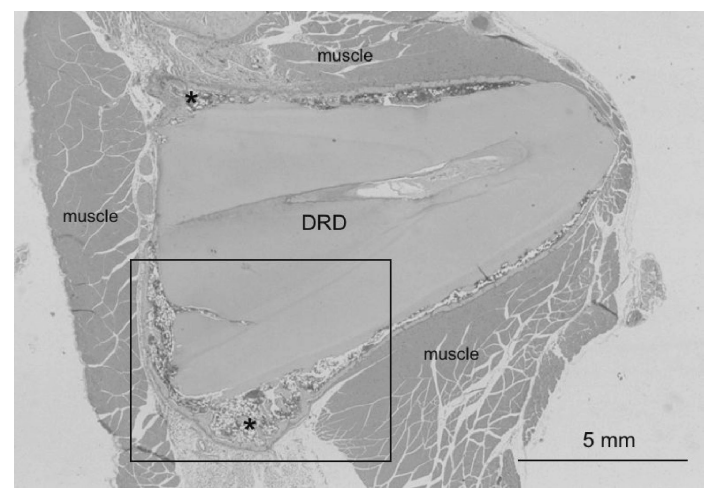

Fig. 7. Histological photograph of decalcified section of BMP-2/DRD at 4 weeks. * Bone marrow on decalcified root (HE).

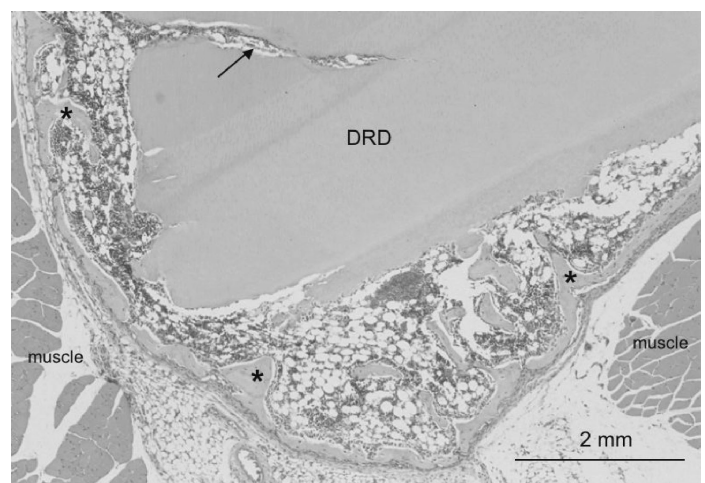

Fig. 8. Higher magnification of $\square$ in Fig. $7 . *$ bone trabeculae, $\uparrow$ cervical crack

\section{Discussion}

Bone morphogenetic proteins (BMPs) are strong accelerating factors with bone-inducing activity. ${ }^{1), 3)}$ Dentin-derived BMPs were extracted with $4 \mathrm{M}$ guanidine $\mathrm{HCl}$, and partially purified from rat, rabbit, and bovine. ${ }^{12-15)}$ The demineralized treatment for bone and dentin increase their osteoinductivity and decrease their antigenesity. ${ }^{16)}$ Even after demineralization, active types of BMPs bind collagen-rich matrices. ${ }^{17}$ ) The decalcified dentin is more absorbable, bone-inducing matrix than the calcified dentin, ${ }^{4)}$ and roll type of decalcified dentin membrane revealed better activity of bone induction. ${ }^{18)}$ Considering these reports, we believe that HAp crystals inhibit release of growth factors as natural BMPs and resist enzyme digestion of dentin collagen, and that the added BMP-2 binds demineralized dentin (DDM, DRD) with both chemical ion-bond and physical adsorption, while natural BMPs binds collagen with covalent bond. In the present study, histological examination showed that the BMP-2/DDM implant and the BMP-2/DRD implant induced bone and marrow, and that DDM granules and DRD mass were partially absorbed (Figs. 4, 6). In the BMP-2/DDM, interconnected bone formation occurred between DDM granules (Fig. 4). On the other hand, in the BMP-2/DRD, bone and marrow were found on the root surface, in the pulp cavity, and in the cracks (Fig. 6). In previous studies, the DDM alone induced bone and cartilage independently, while the DRD alone did not induce bone and cartilage in the mice model at 4 weeks. ${ }^{8), 9)}$ The morphometric analyses demonstrated that the BMP-2/DDM showed $36.3 \%$ in the volume of bone and marrow and $0 \%$ in the volume of cartilage, while the DDM alone showed $1.3 \%$ and $0.4 \%$, respectively at 4 weeks (Table 1). These results indicated that BMP-2 accelerated 
osteoinduction in the granular implant system of DDM. We believe that the granule type and the root type of human dentin will be effective as collagenous carriers of BMP-2 for bone regeneration. Dentin is an acellular collagen-rich tissue, which has bone-inducing and bioabsorbable properties. Especially, autologous dentin has the potential advantages of cell adhesion domain sequences (RGD) and biological recognition. The use of autologous tissues avoids the immune-rejection, and the deleterious side effects of immunosuppressive medications. The autologous dentin collagen is superior to animal-derived materials as medical use. Therefore, human dentin will be able to be recycled in autotransplantation similar to bone autograft for bone regeneration.

\section{Conclusions}

BMP-2 significantly accelerated bone formation in granular and root type carriers of human decalcified dentin. We concluded that human recycled DDM and DRD might be effective biomaterials as carriers of BMP-2 for autologous bone engineering.

Acknowledgments We would like to thank Dr. Masao Ike (Department of Maxillofacial Surgery, Aichi-Gakuin University) very much for great contributions of root-dentin experiments. This work was supported by grants of 2004-5 METI, 2006-7 JST, and 2006-8 Hokkaido, Japan.

\section{References}

1) M. R. Urist, Science, 150, 893-899 (1965).

2) A. H. Reddi and C. B. Huggins, Proc. Soc. Exp. Biol. Med.
Jull., 43, 634-637 (1973).

3) J. M. Wozney, V. Rosen, A. J. Celeste et al., Science, 242, 1528-1534 (1988).

4) J. D. Yeomans and M. R. Urist, Arch. Oral Biol., 12, 999-1008 (1967).

5) G. Bang and M. R. Urist, Arch. Surg., 94, 781-789 (1967).

6) C. Huggins, S. Wiseman and A. H. Reddi, J. Exp. Med., 132, 1250-1258 (1970).

7) M. Murata, D. Sato, T. Akazawa et al., J. Hard Tissue Biol., 11, 110-114 (2003).

8) M. Murata, T. Akazawa, J. Hino et al., Arch. BioCeram. Res., 5, 178-181 (2005).

9) M. Ike, R. Marshall and M. R. Urist, J. Oral Implantol., 24, 124-132 (1998).

10) R. K. Rhodes and E. J. Miller, Biochemistry, 17, 3342-3348 (1978).

11) E. R. Weibel, G. S. Kistler and W. F. Scherle, J. Cell Biol., 30, 23-38 (1966).

12) W. T. Butler, A. Mikulski and M. R. Urist, J. Dent. Res., 56, 228-232 (1977).

13) M. R. Urist, H. Mizutani, M. A. Conover et al., Prog. Clin. Biol. Res., 101, 61-81 (1982).

14) T. Kawai and M. D. Urist, J. Dent. Res., 68, 1069-1074 (1989).

15) K. Bessho, T. Tagawa and M. Murata, J. Oral Maxillofac. Surg., 48, 162-169 (1990).

16) A. H. Reddi, Adv. Med. Phys., 15, 1-17 (1974).

17) M. R. Urist, H. Iwata, P. W. L. Ceccotti et al., Proc. Natl. Acad. Sci. U.S.A., 70, 3511-3515 (1973)

18) T. Inoue, D. A. Deporter and A. H. Melcher, J. Dent. Res., 65, 12-22 (1986) 